Abstract

\title{
Spontaneous Symmetry Breaking Related to the Onset of Instabilities in Low-Temperature Plasma ${ }^{+}$
}

\author{
Dan-Gheorghe Dimitriu 1,* and Maricel Agop ${ }^{2}$ \\ 1 Faculty of Physics, “Alexandru Ioan Cuza” University of Iasi, Iași 700506, Romania \\ 2 Department of Physics, "Gheorghe Asachi” Technical University of Iasi, Iași 700050, Romania; \\ m.agop@yahoo.com \\ * Correspondence: dimitriu@uaic.ro \\ + Presented at Symmetry 2017-The First International Conference on Symmetry, Barcelona, Spain, \\ 16-18 October 2017.
}

Published: 5 January 2018

The study of plasma instabilities is still a hot topic because of their consequences for the development of applications. Here, we report on the experimental investigation and theoretical modeling of the onset of low-temperature plasma instability, as a consequence of the nonlinear dynamics of some complex space charge structures. The obtained results show that the onset of the instability takes place by spontaneous symmetry breaking, which involves transitions between measurable Lie groups. In this situation, a parallel transport in the Levi-Civita sense can be defined, preserving only the direction of the vector and not its modulus. This situation was explained by the phase correlation of the plasma particles.

Acknowledgments: This work was supported by a grant of the Romanian National Council for Scientific Research, CNCS-UEFISCDI, project number PN-III-P4-ID-PCE-2016-0355.

(C) 2018 by the authors. Licensee MDPI, Basel, Switzerland. This article is an open access article distributed under the terms and conditions of the Creative Commons Attribution (CC BY) license (http://creativecommons.org/licenses/by/4.0/). 